\title{
Time-Profile Measurement of an Emulsion Using Multiphoton Ionization Time-of-Flight Mass Spectrometry in Combination with a Microscope
}

\author{
Yosuke SHIMO and Tomohiro UCHIMURA ${ }^{\dagger}$ \\ Department of Materials Science and Engineering, Graduate School of Engineering, University of Fukui, \\ 3-9-1 Bunkyo, Fukui 910-8507, Japan
}

\begin{abstract}
Multiphoton ionization time-of-flight mass spectrometry was applied to the measurement of an oil-in-water emulsion that contained toluene as a dispersed phase. Before the measurement, the sample was sufficiently creamed, and then stirred for a short period of time for dispersion. As a result, several intense spikes appeared on the time profile constructed from the peak area for toluene. In the present study, an optical microscope was used to observe the capillary column for sample introduction, and small toluene droplets along with their aggregates were found in the images of the emulsion flowing through the capillary. The aggregates produced intense spikes comprised of multiple plots, which could be easily marked by applying a moving median filter. In the present study, droplets with minimum diameters as small as $2.9 \mu \mathrm{m}$, which corresponds to $13 \mathrm{fL}$, could be calculated as detectable spikes.
\end{abstract}

Keywords MPI-TOFMS, O/W emulsion

(Received May 6, 2016; Accepted June 20, 2016; Published October 10, 2016)

\section{Introduction}

An emulsion is a system where one liquid is dispersed as small droplets into another liquid, wherein each liquid is immiscible, such as oil and water, where surfactants are often used for emulsification. There are two types of classic emulsions: an oilin-water $(\mathrm{O} / \mathrm{W})$ emulsion where oil is dispersed into water and a water-in-oil (W/O) emulsion where the arrangement is vice versa. Several preparation techniques have been reported, which include the preparation of monodispersed emulsions. ${ }^{1,2}$ Furthermore, multiple emulsions exist, such as a water-in-oil-inwater $(\mathrm{W} / \mathrm{O} / \mathrm{W})$ emulsion and an oil-in-water-in-oil $(\mathrm{O} / \mathrm{W} / \mathrm{O})$ emulsion, and new preparation techniques for controllable monodispersed multiple emulsions have recently been reported. ${ }^{3,4}$ Emulsions are widely applied in academic fields: liquid-liquid extraction or dispersive liquid-liquid microextraction is often used in analytical chemistry ${ }^{5-10}$ and emulsion polymerization is common in polymer chemistry. ${ }^{11,12}$ Emulsions are also commonly used in practical applications, such as foods, drinks, and cosmetics. ${ }^{13}$

Generally, emulsions undergo collapse processes, such as creaming, aggregation, and coalescence, ${ }^{13-15}$ which is different from a normal solution. Therefore, it is not only the stability of emulsions, but also their evaluation methods, that are quite important for use both in fundamental study and in practical application. Several analytical methods for small droplets and/or emulsions have been reported: microscopy, ${ }^{16}$ fluorescence correlation spectroscopy, ${ }^{17}$ fluorescence lifetime imaging, ${ }^{18}$

† To whom correspondence should be addressed.

E-mail: uchimura@u-fukui.ac.jp magnetophoretic velocimetry, ${ }^{19,20}$ high-frequency spectroscopy, ${ }^{21}$ and small-angle X-ray diffraction. ${ }^{22}$ However, direct analysis of the constituents and their concentrations is quite difficult, and these factors influence the stability of emulsions. Mass spectrometry (MS) is a powerful tool for analyzing the constituents of a sample. Electrospray ionization (ESI) is a normal ionization technique that is used for liquid samples, and, until now, surfactant-stabilized picoliter droplets were measured using an ESI quadrupole mass spectrometer (ESI-QMS). ${ }^{23}$ In the case of ESI, however, preparations such as the addition of appropriate solvents are required for the efficient ionization of constituents in a sample, and this changes both the size of the droplets and the stability of the emulsions.

Multiphoton ionization time-of-flight mass spectrometry (MPI-TOFMS) is a mass analytical method with optical selectivity and robustness against contamination. ${ }^{24-35}$ Although this is the method that is normally applied to a gas sample, we recently reported a method based on MPI-TOFMS for the evaluation of emulsions with no pretreatment. ${ }^{36}$ Briefly, a sample-introduction system using a pair of concentric capillaries was developed, where an emulsion was directly passed through the inner capillary. Analyte species were then vaporized in a vacuum chamber; these were subsequently ionized by MPI using an ultraviolet laser. In that report, $\mathrm{O} / \mathrm{W}$ emulsions containing toluene as a dispersed phase were measured, and a time profile of the peak areas for toluene was constructed from a series of mass spectra. As a result, several spikes in the time profile were found when measuring an emulsion with white turbidity. The occurrence of spikes was probably due to the presence of small toluene droplets in the emulsion, which were vaporized and therefore highly concentrated toluene molecules were simultaneously ionized via MPI. Moreover, the signal 
intensities of toluene were found to have decreased with time during monitoring of the lower portions of an emulsion, which is where the creaming gradually occurred. Furthermore, we applied this method to our evaluation of hydrophobic and hydrophilic compounds and their extraction behavior toward oil droplets in $\mathrm{O} / \mathrm{W}$ emulsions, ${ }^{37}$ along with our analysis of the constituents that exist in $\mathrm{W} / \mathrm{O} / \mathrm{W}$ multiple emulsions. ${ }^{38}$

For inquiries such as this, this technique confers a distinguishing ability to monitor a local microenvironment in an emulsion. However, the condition of small droplets flowing through a capillary column during sample introduction has not yet been confirmed. To understand the relationship between the diameter of small droplets and the resultant signal intensities of spikes, the minimum size of a small droplet that can be detected as a spike is particularly important when applying this method to emulsion analysis. In the present study, the toluene $\mathrm{O} / \mathrm{W}$ emulsion flowing through the capillary column was observed using an optical microscope, and the obtained results were studied together with the results of the time profile obtained by MPI-TOFMS. Moreover, we proposed a method that uses a moving median filter ${ }^{39,40}$ to evaluate whether each spike is comprised of single or multiple plots, and also discussed spikes comprised of multiple plots from the point of view of aggregates of the small droplets. Furthermore, we calculated the minimum diameter of a toluene droplet that can be detected as a spike.

\section{Experimental}

\section{Reagents and sample preparation}

We prepared an $\mathrm{O} / \mathrm{W}$ emulsion with white turbidity in the present study. Samples of toluene and sodium dodecyl sulfate (SDS) were purchased from Wako (Japan), and were used as a dispersed phase and a surfactant, respectively. First, SDS was added to $50 \mathrm{~mL}$ of distilled water in a sample container, and the solution was shaken manually until the SDS could not be visualized. Toluene was then added and a cap was placed on the sample container, and then the container was sonicated for $1 \mathrm{~min}$. The final concentrations of toluene and SDS were 5900 and $3100 \mathrm{ng} / \mu \mathrm{L}$ of water. The height of the obtained $\mathrm{O} / \mathrm{W}$ emulsion in the sample container was $c a .6 \mathrm{~cm}$. The emulsion with white turbidity was left sitting for 1 day before any measurement, so that the creaming could sufficiently proceed; as a result, almost all of the lower portions of the sample seemed to be visually transparent.

\section{Apparatus}

Figure 1 shows a schematic diagram of the experimental apparatus. Details of the MPI-TOFMS used in the present study were reported elsewhere, ${ }^{36}$ and are briefly described here. The inner/outer diameters of the inner and outer columns for a pair of concentric capillaries for sample introduction were 25/150 and $320 / 450 \mu \mathrm{m}$, respectively, and the lengths of the inner and outer columns were 40 and $120 \mathrm{~cm}$, respectively. The position of the nozzle tip of the inner column was adjusted $c a .3 \mathrm{~mm}$ inward from that of the outer column. The temperature of the nozzle tip was set at $40^{\circ} \mathrm{C}$. The end of the inner column opposite the nozzle was inserted into a sample container through a small hole (diameter $3 \mathrm{~mm}$ ) in the cap. The emulsion was introduced via the inner column, and ambient air was introduced via the outer column. The pressure of the TOFMS was typically $1 \times 10^{-2} \mathrm{~Pa}$. The length of the inner column from the tip in the sample container to the observation point of an optical microscope was set at $40 \mathrm{~cm}$, and that from the observation point to the nozzle tip was $80 \mathrm{~cm}$.

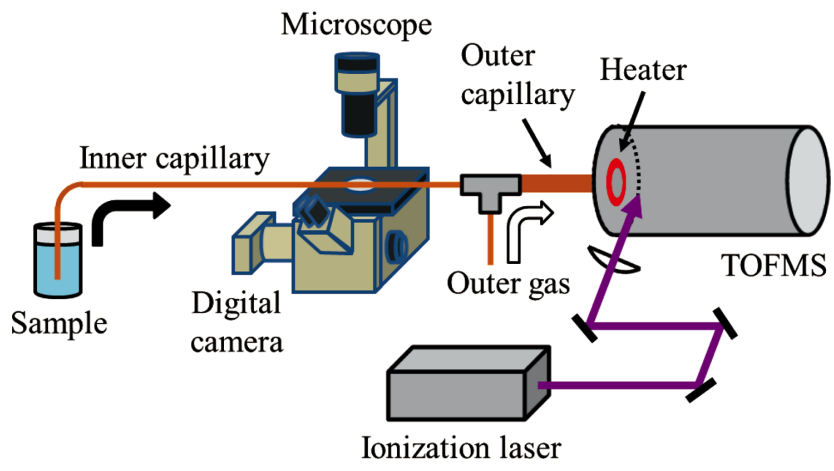

Fig. 1 Schematic diagram of the apparatus.

The fourth-harmonic emission of a Nd:YAG laser (Rayture Systems, GAIA II, $266 \mathrm{~nm}, 10 \mathrm{~Hz}, 4 \mathrm{~ns}$ ) was employed for MPI. The laser beam was focused via a plano-convex lens with a focal length of $200 \mathrm{~mm}$. A linear-type TOFMS was used, which is now commercially available (Hikari-GK, HGK-1, drift length $60 \mathrm{~cm}$, Fukuoka, Japan). The distance between the tip of the outer column and the ionization point was adjusted to $2 \mathrm{~mm}$. A series of mass spectra were recorded using a digitizer (Acqiris/ Agilent Technologies, AP240; sampling rate, $1 \mathrm{GS} / \mathrm{s}$; bandwidth, $1 \mathrm{GHz}$ ). The laser beam for ionization was not introduced for the first $30 \mathrm{~s}$ after starting the recording by the digitizer in order to determine a background (zero) level. The present study involved extended durations of time for measurement, which could have resulted in the acquisition of huge amounts of data that would have been difficult to process. Therefore, we recorded the data for every $600 \mathrm{~s}$, and then immediately restarted the recording, which resulted in a time interval of a few seconds between each recording. The time profile for toluene was constructed by extracting the peak areas of both molecular ions $(\mathrm{m} / \mathrm{z}, 92)$ and fragment ions $(\mathrm{m} / \mathrm{z}, 91)$ from a series of obtained mass spectra. Moreover, the time profiles filtered via a moving median filter with a 3-point window were indicated for evaluating the obtained spikes, which will be described in detail later. The data processing was performed via a program generated in-house using graphical language (National Instruments, LabVIEW).

An upright microscope (Olympus, IX51, 10×, NA 0.25) equipped with a digital camera (Nikon, Digital Sight DS-U1) was placed between the sample container and the TOFMS, and a video of the emulsion flowing through the capillary column was recorded. Although the measurement of the emulsion using MPI-TOFMS and the recording of the video were performed simultaneously, the obtained bright-field images were, unfortunately, unfocused. Therefore, the images shown in Fig. 2 were re-recorded another day.

\section{Results and Discussion}

A time profile for toluene and images of the emulsion flowing through the capillary column

Figure 2 shows the time profile for toluene in an $\mathrm{O} / \mathrm{W}$ emulsion obtained by using MPI-TOFMS. The bright-field images of the emulsion flowing through the capillary column observed at 150, 231, 443, 1010, and $6300 \mathrm{~s}$ after stirring are also shown in this figure.

As described above, the emulsion was left standing for 1 day before the measurement so that the creaming sufficiently 

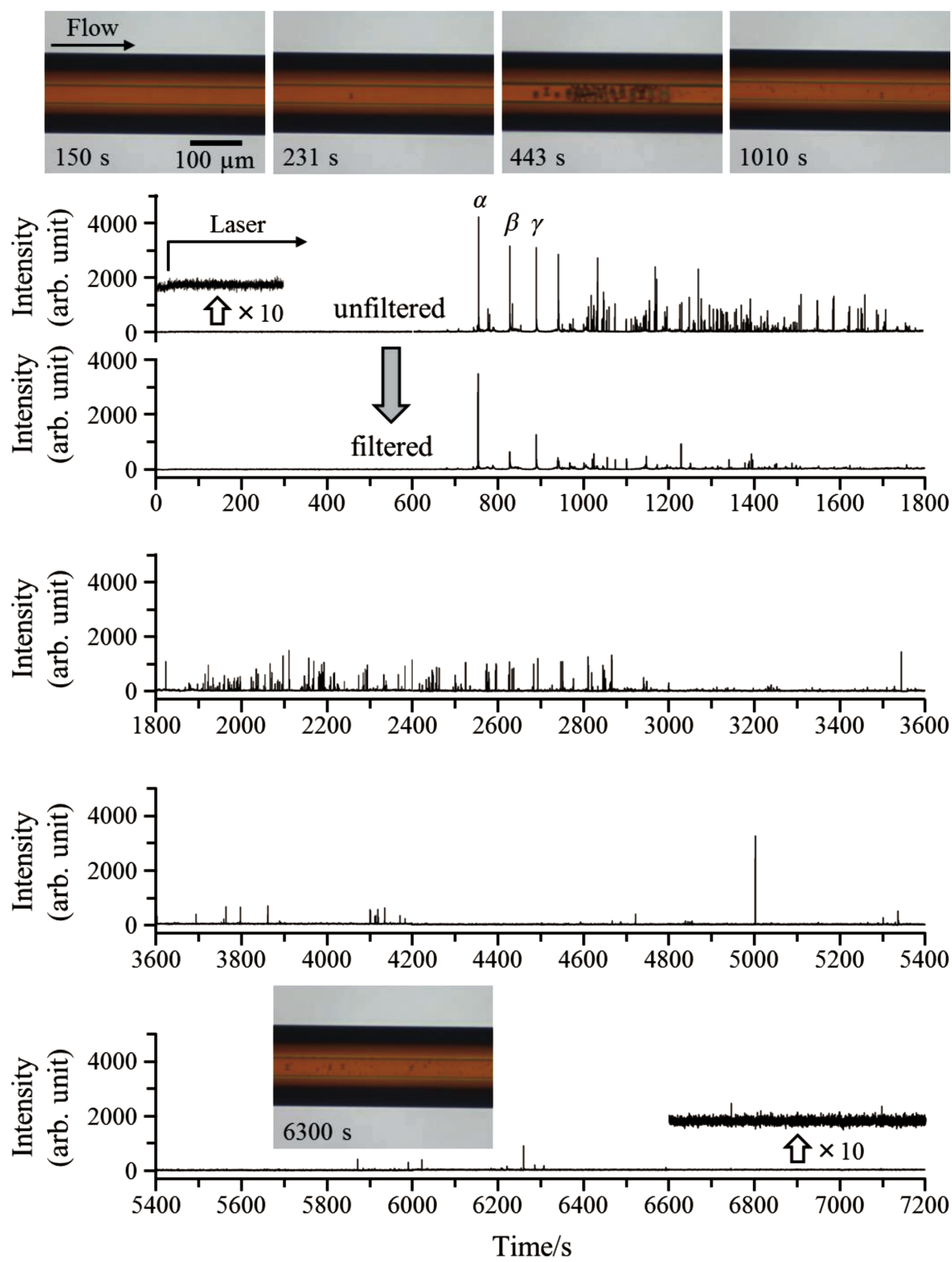

Fig. 2 Time profile for toluene in an O/W emulsion measured by MPI-TOFMS. Stirring was started at $0 \mathrm{~s}$ (see text). The ionization laser was introduced $30 \mathrm{~s}$ after starting the recording. Bright-field images are also shown; the times obtained by a microscope are indicated in each image. The time profile for $0-1800 \mathrm{~s}$ after applying a moving median filter with a 3 point window is also shown. The peaks specified by $\alpha, \beta$, and $\gamma$ were used for calculating $S_{\max }$ (see text).

proceeded, after which the inner column for sample introduction was inserted into a sample container. The tip of the capillary was set $1.5 \mathrm{~cm}$ from the bottom of the sample container, where it was visually transparent. After inserting the capillary column, the condition was maintained for $c a .1 \mathrm{~h}$ in order to ensure that the sample had completely filled, and was flowing through the capillary. Then, the stirring of the emulsion and the recording of a series of mass spectra were simultaneously begun. The sample was sonicated for $15 \mathrm{~s}$, and the upper portion of the emulsion had a white turbidity similar to that of a mist. After being sonicated, the sample was shaken manually for $7-8$ s so that toluene was dispersed entirely into the sample container. Although creaming re-occurred after the shaking was stopped, the sample was translucent rather than transparent when the measurement in Fig. 2 was finished.

From the time profile shown in Fig. 2, signals with small intensities were detected just after the laser beam for ionization was introduced. As described later, the line velocity of the sample was ca. $1-2 \mathrm{~mm} / \mathrm{s}$ and, therefore, a transparent part of the sample after sufficient creaming could still be monitored at the beginning of the measurement, where small droplets were not observed, such as with the image taken at $150 \mathrm{~s}$. Then, small droplets were observed $c a$. $200-240 \mathrm{~s}$ after stirring, such as in the image taken at $231 \mathrm{~s}$. After that, large aggregates of small droplets were confirmed, such as the image taken at $443 \mathrm{~s}$. Both aggregates and separated small droplets, as in the image taken at $1010 \mathrm{~s}$, could be found for a certain period of time, but both the size and the frequency of the appearance of aggregates seemed to decrease with time. These phenomena were considered to be related to creaming of the emulsion.

Several intense spikes in the time profile probably arose from the aggregates of the small droplets between 760 and $1300 \mathrm{~s}$. In addition, spikes with moderately strong intensities were intermittently detected at between 680 to $2870 \mathrm{~s}$, and were 
probably caused by the separated small droplets. The frequency of the appearance of spikes was decreased $2870 \mathrm{~s}$ after stirring. Finally, only a few small spikes appeared on the time profiles at $6600-7200 \mathrm{~s}$. At that time, a flow of small droplets with a diameter of a few $\mu \mathrm{m}$ could be observed, such as the image taken at $6300 \mathrm{~s}$, although the exact size was difficult to determine because it was rather small and the focus was often mismatched due to the longitudinal width of the capillary column. Incidentally, a few small droplets were confirmed to have adhered onto the inner wall of the inner capillary column at least $1800 \mathrm{~s}$ after stirring, and a small number was added with time. We could not confirm the presence of adhered droplets during the passage of large aggregates, and, therefore, such aggregates might have removed the adhered droplets from the inner wall of the capillary.

During the measurement, the line velocities of the small droplets ( $c a .1-2 \mathrm{~mm} / \mathrm{s}$ ) were derived from the results of the video, although they differed from one another. The obtained line velocities were consistent at $c a .1 .6 \mathrm{~mm} / \mathrm{s}$, which was calculated from the results of the time interval ( $c a .480 \mathrm{~s}$ ) between the detection of the most intense spike ( $c a .760 \mathrm{~s}$, specified by $\alpha$ in Fig. 2) and the simultaneous observation of a large aggregate $(c a .280 \mathrm{~s})$ that caused the intense spike, although the images were unfocused, and the length between the observation point of the optical microscope and the position of the nozzle tip of the inner column was $80 \mathrm{~cm}$.

In the present study, large aggregates of small toluene droplets were considered to produce the intense spikes described above. In this case, highly concentrated toluene should be almost continuously introduced into a TOFMS for a certain period of time, and, therefore, the spikes should be comprised of multiple plots, rather than a single plot. However, the procedure for checking spikes one-by-one is tedious and time-consuming. Therefore, a moving median filter was applied to the data analysis of the time profile to temporarily confirm whether each spike was comprised of single or multiple plots. Briefly, a moving median filter is a digital filtering technique where each value in a series of data is replaced by the median of the neighboring data. A moving median filter is normally used to remove spike noise, ${ }^{39,40}$ e.g., a spike comprised of a single plot can be removed by applying a moving median filter with a 3-point window. In other words, if spikes still exist after applying a moving median filter, we can estimate that the spikes existing before filtering would be comprised of multiple plots, although at least one exception would be the data of spikes and non-spikes appearing alternately on the original time profile. The filtered time profile from $0-1800 \mathrm{~s}$ is also shown in Fig. 2. We could easily confirm a tendency whereby intense spikes before filtering continued to exist as spikes after filtering, while spikes other than intense ones were considerably eliminated. For example, the three intense spikes specified by $\alpha, \beta$, and $\gamma$ were also apparently confirmed as spikes after filtering. Therefore, these spikes before filtering were found to be comprised of multiple plots. Particularly, the peak specified by $\alpha$ was comprised of three plots with large signal intensities. In this manner, we can easily mark the spikes that are comprised of multiple plots, which probably arose from the aggregates of small droplets. Incidentally, aggregates observed in the present study were considered to not have been generated in the sample container, but to have been gradually generated as they flowed through the capillary column due to the differences in the line velocities among the small droplets. In particular, in order to set a microscope between the sample container and the TOFMS, the length of the inner capillary column in the present study was rather long $(120 \mathrm{~cm})$ compared with that in the previous report
$(40 \mathrm{~cm}),{ }^{36,37}$ and this resulted in promoting the generation of aggregates during flow through the capillary column during sample introduction.

\section{The minimum size of a droplet that will elicit a detectable spike}

From the obtained time profile shown in Fig. 2, we deduced the minimum diameter of a small toluene droplet $\left(D_{\min }\right)$ that could flow through a capillary column and subsequently cause a detectable spike on the time profile.

The first consideration must be the relationship between the diameter of a small droplet $(D)$ in the capillary column and the signal intensity of the spike $(S)$ derived from the corresponding droplet. In this technique, the velocity of the analyte species passing through the ionization point is considerably high (several hundreds of $\mathrm{m} / \mathrm{s}$, deduced from the previous report),${ }^{41}$ compared with the line velocity of the small droplet flowing through the capillary column $(1-2 \mathrm{~mm} / \mathrm{s})$, i.e., the velocity was increased $c a$. $10^{5}$. Therefore, with respect to the axis of the sample flow, the analyte species exists in the ionization region more widely by far than it does in the capillary column. Meanwhile, the ionization beam waist was very small (roughly $10 \mu \mathrm{m}$ ); therefore, the beam irradiated a small portion of the analyte species in TOFMS. In other words, the beam can be considered to pass through the thin section of the small droplet perpendicular to the direction with respect to the axis of the sample flow. Hence, the signal intensity of the spike can be considered to be directly proportional to the square of the diameter of the small droplet, i.e., $S$ is proportional to $D^{2}$. However, if a droplet was quite small, e.g., the diameter was less than $c a .0 .1 \mathrm{~nm}\left(=10 \mu \mathrm{m} / 10^{5}\right)$, the volume of the small droplet would be directly proportional to the cubes of its diameter, but such droplets need not be taken into consideration. Therefore, $D_{\min }$ can be estimated using the following equation:

$$
D_{\min }=D_{\max } \sqrt{\frac{S_{\min }}{S_{\max }}}
$$

where $S_{\min }$ and $S_{\max }$ are the minimum and maximum signal intensities of the spikes that appear in the obtained time profile; $D_{\max }$ is the diameter of the small toluene droplet, which causes the maximum intensity of the spike, $S_{\max }$. The $S_{\max }$ spike arises from a droplet and/or an aggregate that fulfills the inner column. In the present study, $D_{\max }=25 \mu \mathrm{m}$ was applied as the diameter of the inner capillary column for the sample introduction. The value of $S_{\max }$ can be easily obtained by the results shown in Fig. 2, but the deviation of the signal intensities should be taken into account. In the present study, $S_{\max }=3500$ was used from the average of the signal intensities of 3 spikes, which are indicated by $\alpha, \beta$, and $\gamma$ in Fig. 2.

Next, the background fluctuation, $B$, was determined to be twice the standard deviation of the signal intensities other than the spikes when introducing the laser beam for ionization. In the present study, $B=16$ was obtained from the result when measuring the transparent part of the emulsion. Therefore, we estimated that a spike with an $S / B=219$ would be generated from a droplet with a diameter of $25 \mu \mathrm{m}$. Assuming that a spike with an $S / B=3$ was defined as detectable, an $S_{\min }=48$ would be obtained. As a result, the minimum diameter of a small toluene droplet, $D_{\min }$, was calculated to be $2.9 \mu \mathrm{m}$ using Eq. (1), which corresponds to $13 \mathrm{fL}$. The calculated diameter was appropriate, when compared with images obtained at the final stage of measurement, although the size was again somewhat difficult to reference. 


\section{Conclusions}

In the present study, a toluene $\mathrm{O} / \mathrm{W}$ emulsion flowing through a capillary column for a sample introduction of MPI-TOFMS was observed via a microscope, and obtained images were compared with the results of the time profile for toluene, as measured by MPI-TOFMS. As a result, intense spikes comprised of multiple plots were suggested to have arisen from the aggregates of the small toluene droplets. We also demonstrated that the use of a moving median filter was helpful in judging whether spikes were comprised of single or multiple plots. Moreover, the minimum size of a detectable droplet as a spike, $c a .3 \mu \mathrm{m}$, could be calcutated. Further small droplets, e.g., with a diameter of $1 \mu \mathrm{m}$ if needed, might be detectable by using the inner capillary column with smaller diameters, because the volume of fluid other than droplets can be reduced, which should result in reducing the standard deviation of the signal intensities other than the spikes.

Unfortunately, the observed aggregates probably occurred during flow through the capillary column, due to using the relatively long capillary column for the setting of a microscope. In other words, MPI-TOFMS combined with a microscopic system for monitoing the sample introduction port is required and is surely useful in detailed evaluations of several types of emulsions of not only $\mathrm{O} / \mathrm{W}$ and $\mathrm{W} / \mathrm{O}$ emulsions, but also for multiple emulsions.

\section{Acknowledgements}

This work was supported by a research grant provided by the JGC-S Scholarship Foundation.

\section{References}

1. F. Leal-Galderon, V. Schmitt, and J. Bibette, "Emulsion Science Basic Principles", 2nd ed., 2007, Springer, New York.

2. H. Tanaka, S. Yamamoto, A. Nakamura, Y. Nakashoji, N. Okura, N. Nakamoto, K. Tsukagoshi, and M. Hashimoto, Anal. Chem., 2015, 87, 4134.

3. L.-Y. Chu, A. S. Utada, R. K. Shah, J.-W. Kim, and D. A. Weitz, Angew. Chem. Int. Ed., 2007, 46, 8970.

4. D. Liu, B. Hakimi, M. Volny, J. Rolfs, X. Chen, F. Turecek, and D. T. Chiu, Anal. Chem., 2013, 85, 6190.

5. N. Yanase, H. Naganawa, T. Nagano, and J. Noro, Anal. Sci., 2011, 27, 171.

6. J. Regueiro, M. Llompart, C. Garcia-Jares, J. C. GarciaMonteagudo, and R. Cela, J. Chromatogr. A, 2008, 1190, 27.

7. M. Saraji and A. A. H. Bidgoli, Anal. Bioanal. Chem., 2010, 397, 3107.

8. T. Hayama, Y. Yabuuchi, T. Iwamatsu, E. Tamashima, Y. Kawami, M. Itoyama, H. Yoshida, M. Yamaguchi, and H. Nohta, Talanta, 2013, 117, 27.

9. L. Guo and H. K. Lee, Anal. Chem., 2014, 86, 3743.

10. M.-I. Leong, M.-R. Fuh, and S.-D. Huang, J. Chromatogr.
A, 2014, 1335, 2.

11. W. D. Harkins, J. Am. Chem. Soc., 1947, 69, 1428.

12. C. S. Chern, Prog. Polym. Sci., 2006, 31, 443.

13. K. Holmberg, B. Jönsson, B. Kronberg, and B. Lindman, "Surfactants and Polymers in Aqueous Solution", 2nd ed., 2003, John Wiley \& Sons, Chichester.

14. E. Dickinson, M. Golding, and M. J. W. Povey, J. Colloid Interface Sci., 1997, 185, 515.

15. R. Chanamai and D. J. McClements, Colloids Surf., 2000, $172,79$.

16. S. Tsukahara, Y. Shishino, and T. Fujiwara, Langmuir, 2011, 27, 7392.

17. N. Pal, S. D. Verma, M. K. Singh, and S. Sen, Anal. Chem., 2011, 83, 7736.

18. X. C. i Solvas, M. Srisa-Art, A. J. deMello, and J. B. Edel, Anal. Chem., 2010, 82, 3950.

19. M. Suwa and H. Watarai, Anal. Chem., 2001, 73, 5214.

20. Y. Iiguni and H. Ohtani, Anal. Sci., 2013, 29, 35.

21. K. Kageshima, T. Takei, and Y. Sugitani, Anal. Sci., 2003, 19, 757.

22. S. Arima, S. Ueno, A. Ogawa, and K. Sato, Langmuir, 2009, 25, 9777.

23. C. A. Smith, X. Li, T. H. Mize, T. D. Sharpe, E. I. Graziani, C. Abell, and W. T. S. Huck, Anal. Chem., 2013, 85, 3812.

24. R. Zenobi, J.-M. Philippoz, P. R. Buseck, and R. N. Zare, Science, 1989, 246, 1026.

25. J. Matsumoto, C.-H. Lin, and T. Imasaka, Anal. Chim. Acta, 1997, 343, 129.

26. T. Uchimura and T. Imasaka, Anal. Chem., 2000, 72, 2648.

27. R. Zimmermann, W. Welthagen, and T. Gröger, $J$. Chromatogr. A, 2008, 1184, 296.

28. A. M. Rijs, B. O. Crews, M. S. de Vries, J. S. Hannam, D. A. Leigh, M. Fanti, F. Zerbetto, and W. J. Buma, Angew. Chem. Int. Ed., 2008, 47, 3174.

29. B. K. Gullett, L. Oudejans, D. Tabor, A. Touati, and S. Ryan, Environ. Sci. Technol., 2012, 46, 923.

30. K. Ohishi, T. Sakamoto, J. Saikawa, N. Ishigaki, K. Tojo, Y Ido, S. Hayashi, S. Ishiuchi, K. Misawa, and M. Fujii, Anal. Sci., 2013, 29, 291.

31. S. Sakurai and T. Uchimura, Anal. Sci., 2014, 30, 891.

32. K. Titze, T. Zollitsch, U. Heiz, and U. Boesl, Chem. Phys. Chem., 2014, 15, 2762.

33. A. Hamachi, T. Okuno, T. Imasaka, Y. Kida, and T. Imasaka, Anal. Chem., 2015, 87, 3027.

34. T. Fujii and T. Uchimura, Anal. Methods, 2015, 7, 2611.

35. J. Kleeblatt, B. Stengel, C. Radischat, J. Passig, T. Streibel, O. Sippula, R. Rabe, H. Harndorf, and R. Zimmermann, Anal. Methods, 2015, 7, 3608.

36. H. Ishigami, Y. Tsuda, and T. Uchimura, Anal. Methods, 2014, 6, 5615.

37. H. Fukaya, Y. Tsuda, and T. Uchimura, Anal. Methods, 2016, 8, 270.

38. Y. Tsuda and T. Uchimura, Anal. Sci., 2016, 32, 789.

39. C. L. do Lago, V. F. Juliano, and C. Kascheres, Anal. Chim. Acta, 1995, 310, 281.

40. C. A. Hastings, S. M. Norton, and S. Roy, Rapid Commun. Mass Spectrom., 2002, 16, 462.

41. G. Tokumoto, H. Saburi, S. Miyagawa, and T. Uchimura, Bunseki Kagaku, 2013, 62, 595. 